\title{
Systemic transplantation of adult multipotent stem cells prevents articular cartilage degeneration in a mouse model of accelerated ageing
}

\author{
Seth D. Thompson ${ }^{1,2,3+}$, Rajeswari Pichika ${ }^{1,2+}$, Richard L. Lieber ${ }^{1,2}$ and Mitra Lavasani ${ }^{1,2,3^{*}}$ (D)
}

\begin{abstract}
Background: Osteoarthritis $(\mathrm{OA})$ is one of the most prevalent joint diseases of advanced age and is a leading cause of disability worldwide. Ageing is a major risk factor for the articular cartilage (AC) degeneration that leads to OA, and the age-related decline in regenerative capacity accelerates OA progression. Here we demonstrate that systemic transplantation of a unique population of adult multipotent muscle-derived stem/progenitor cells (MDSP Cs), isolated from young wild-type mice, into $\mathrm{Zmpste}^{4^{-/-}}$mice (a model of Hutchinson-Gilford progeria syndrome, a condition marked by accelerated ageing), prevents ageing-related homeostatic decline of AC.

Results: MDSPC treatment inhibited expression of cartilage-degrading factors such as pro-inflammatory cytokines and extracellular matrix-proteinases, whereas pro-regenerative markers associated with cartilage mechanical support and tensile strength, cartilage resilience, chondrocyte proliferation and differentiation, and cartilage growth, were increased. Notably, MDSPC transplantation also increased the expression level of genes known for their key roles in immunomodulation, autophagy, stress resistance, pro-longevity, and telomere protection. Our findings also indicate that MDSPC transplantation increased proteoglycan content by regulating chondrocyte proliferation.

Conclusions: Together, these findings demonstrate the ability of systemically transplanted young MDSPCs to preserve a healthy homeostasis and promote tissue regeneration at the molecular and tissue level in progeroid AC. These results highlight the therapeutic potential of systemically delivered multipotent adult stem cells to prevent age-associated AC degeneration.
\end{abstract}

Keywords: Progeria, Accelerated ageing, Articular cartilage, Adult stem cells, Transplantation, Regenerative medicine

\footnotetext{
* Correspondence: mlavasani@sralab.org

${ }^{\dagger}$ Seth D. Thompson and Rajeswari Pichika contributed equally to this study.

'Shirley Ryan Abilitylab (Formerly the Rehabilitation Institute of Chicago), 355

E. Erie St, IL 60611 Chicago, USA

${ }^{2}$ Department of Physical Medicine and Rehabilitation, Northwestern

University, Chicago, USA

Full list of author information is available at the end of the article
}

C C The Author(s). 2021 Open Access This article is licensed under a Creative Commons Attribution 4.0 International License, which permits use, sharing, adaptation, distribution and reproduction in any medium or format, as long as you give appropriate credit to the original author(s) and the source, provide a link to the Creative Commons licence, and indicate if changes were made. The images or other third party material in this article are included in the article's Creative Commons licence, unless indicated otherwise in a credit line to the material. If material is not included in the article's Creative Commons licence and your intended use is not permitted by statutory regulation or exceeds the permitted use, you will need to obtain permission directly from the copyright holder. To view a copy of this licence, visit http://creativecommons.org/licenses/by/4.0/. The Creative Commons Public Domain Dedication waiver (http://creativecommons.org/publicdomain/zero/1.0/) applies to the data made available in this article, unless otherwise stated in a credit line to the data. 


\section{Background}

Ageing-related degeneration of the knee articular surface, degradation of aggrecans, and loss of matrix tensile strength and stiffness [1, 2] results in osteoarthritis (OA), the most prevalent and debilitating joint disease of advanced age and the leading cause of disability in older adults worldwide [3, 4]. Ageing results in a disruption of cartilage homeostasis. Accumulation of catabolic factors-such as pro-inflammatory cytokines, senescenceassociated secretory phenotype (SASP) factors [5], and matrix metalloproteinases (MMPs) alters the tissue microenvironment, contributes to oxidative stress, and can augment inflammatory responses $[6,7]$. With increasing age, chondrocyte density and responsiveness to proliferative and anabolic factors are reduced $[8,9]$. Because articular chondrocytes rely on autophagy as the primary mechanism for maintaining healthy function and survival [10], the gradual decrease of chondrocyte autophagic activity during ageing induces senescence, ultimately increasing OA severity [11].

While tissue- and cellular-level changes in aged cartilage have been characterized [12], an effective therapy or preventative treatment for age-related articular cartilage (AC) degeneration has not been developed. Current cellular treatments, including autologous chondrocyte implantation, autologous matrix-induced chondrogenesis (AMIC), and intra-articular injection of mesenchymal stem cells, have been effective for small, mainly injuryinduced articular cartilage loss [13-16]; however, their effectiveness is questionable in cases of cartilage degradation, rheumatic disease, and considerable restriction of joint mobility, which substantially limits their applicability for the treatment of ageing-related changes [17].

We have isolated a unique population of adult multipotent stem cells, muscle-derived stem/progenitor cells (MDSPCs), from skeletal muscle via a modified preplate technique [18]. MDSPCs have the capacity for long-term proliferation, self-renewal, and multi-lineage differentiation, and are resistant to oxidative stress, all of which likely contribute to their ability to promote regeneration [19-23]. MDSPCs have also been shown to undergo chondrogenic differentiation in vitro and to repair cartilage defects as efficiently as chondrocytes [23], after local transplantation in a young host, injury-induced OA model [24]. Previously, we established that MDSPCs isolated from naturally aged and progeroid (i.e., accelerated ageing) mice showed a reduction in stemness capacity, including proliferation and differentiation; however, these phenotypic impairments were rescued by coculture with, or by conditioned media from young MDSPCs [22, 25]. In addition, we previously demonstrated that systemic transplantation of young MDSPCs into a progeroid mouse model delayed the onset of multiple age-related pathologies, leading to a doubling of lifespan and a significant extension of healthspan [22]. Our recently published results indicate that systemic transplantation of young MDSPCs into naturally aged (2-yr old) mice restores aging $\mathrm{AC}$ - at the molecular, tissue, and functional levels-highlighting their therapeutic ability to reverse age-related osteoarthritis [26]. Together, these observations strongly suggest that MDSPCs exert a therapeutic effect on the aged microenvironment by systemically-acting secreted factors.

Zmpste $24^{-/-}$mice are an established murine model of Hutchinson-Gilford progeria syndrome (HGPS), which features many musculoskeletal degenerative changes similar to those of advanced ageing [27-30]. HGPS is manifested predominantly in the connective tissue, with the most prominent histological changes observed in the cartilage, bone, skin, and cardiovascular tissues [31]. Here, we report that systemic transplantation of MDSP $\mathrm{Cs}$ rejuvenates progeroid $\mathrm{AC}$ at the molecular and tissue levels in $Z$ mpste $24^{-/-}$mice.

\section{Results}

Articular cartilage of progeroid mice shows ageingrelated imbalances associated with local catabolic and anabolic activity

To identify age-associated articular cartilage (AC) changes in progeroid mice, gene expression in knee joints of 5-6 month-old Zmpste $24^{-/-}$mice was compared to that of age-matched wild-type (WT) littermates. Quantitative reverse transcriptase polymerase chain reaction (qRT-PCR) was used to assess expression of genes encoding pro-inflammatory cytokines, extracellular matrix (ECM) proteinases, and ECM components (Fig. 1). In the AC of progeroid mice, we observed a statistically significant increase in Illa, Il6, and Tnf-proinflammatory factors associated with the senescenceassociated secretory phenotype (SASP)-compared to WT littermates (Fig. 1a). Gene expression of $M m p 3$, Mmp13, and Adamts5-catabolic factors involved with cleaving ECM components such as collagen type II and aggrecans, [32-34] were also significantly increased in Zmpste $24^{-/-}$progeroid mice (Fig. 1b). In addition, we observed a significant increase in the expression level of Col1a1, responsible for cartilage tensile strength and stiffness, in the articular cartilage of Zmpste $24^{-1-}$ progeroid mice compared to WT littermates; however, the expression level of pro-regenerative matrix cellular components such as Col2a1, Acan, Vcan, and Bgn were not significantly altered (Fig. 1c). Of note, we did not observe a significant difference in telomere protection (Pot1b) or autophagy suppression (Mtor) genes in Zmpste $24^{-/-}$progeroid mice compared to WT mice (Fig. 1d). Furthermore, the antioxidant response gene $(G p x)$ was significantly increased in progeroid mice compared to WT mice, while no change was observed in 
a

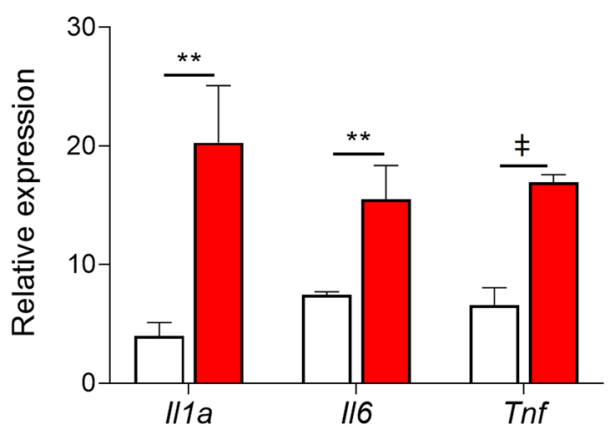

b

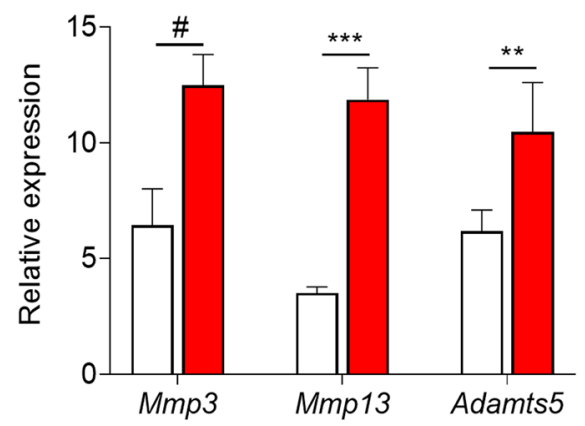

C
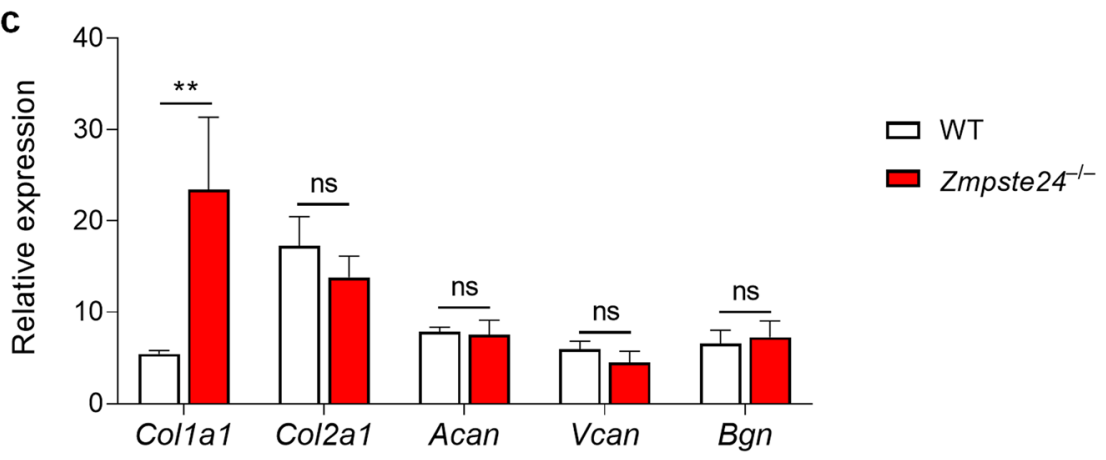

d

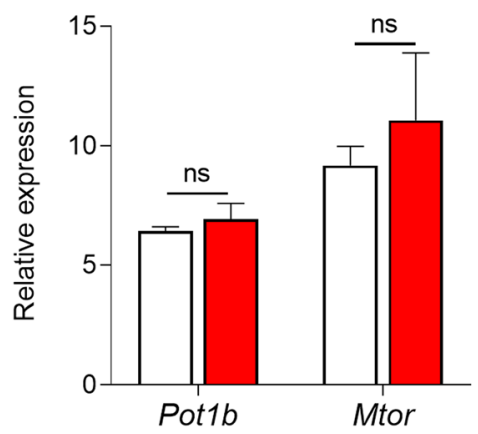

e

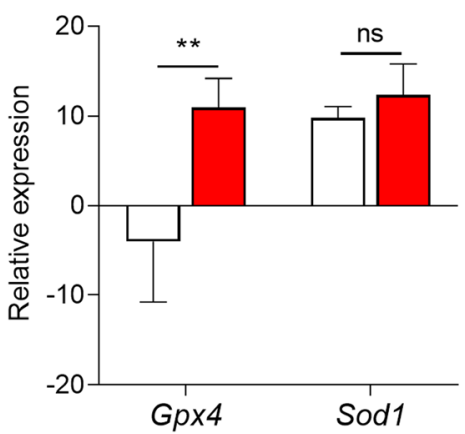

Fig. 1 Alteration in Gene Expression Profile of Articular Cartilage in Zmpste24-deficient (Zmpste24-1-) Progeroid Mice. Articular cartilage from 5-6 month-old adult wild type $(n=6)$ and age-matched Zmpste24-/- littermate knee joints $(n=5)$ were analyzed for the mRNA expression level of $(\mathbf{a}-$ c genes encoding pro-inflammatory cytokines and senescence-associated secretory phenotype (SASP) factors (II1a, Il6, and Tnf), extracellular matrix (ECM) proteinases (Mmp3, Mmp13, and Adamts5), ECM components (Col1a1, Col2a1, Acan, Vcan, and Bgn) using qRT-PCR. d-e Genes associated with telomere protection (Pot1b), autophagy suppression (Mtor), antioxidant response (Gpx4), and oxidative stress-response (Sod1). Expression values are relative to housekeeping genes, Gapdh and Hmgb1. Data are mean \pm SEM. ${ }^{* *} p<0.01,{ }^{* * *} p<0.001,{ }^{\#} p<0.0001,{ }^{*} p<$ 0.000001 , ns: not significant using two-tailed, unpaired Student's $t$-test or Welch's unequal variance $t$-test

oxidative stress-response (Sod1) expression. (Fig. 1e). Taken together, these findings demonstrate a catabolicanabolic imbalance in the AC of progeroid Zmpste24-1mice.

\section{Systemic transplantation of young MDSPCs preserves} healthy articular cartilage homeostasis in progeroid mice To determine whether the loss of AC homeostasis due to accelerated ageing in Zmpste $24^{-1-}$ mice can be prevented, we investigated the effect of systemic transplantation of young MDSPCs on the AC microenvironment.
Littermate pairs of Zmpste $24^{-/-}$mice were injected intraperitoneally (IP) with $2 \times 10^{5}$ young MDSPCs per gram of body weight (Z-IP) or an equal volume of PBS (Z-PBS) at 2 months of age and again at 4 months of age (Fig. 2a). The AC from the knee joints was processed at 5-6 months of age and gene expression was measured using qRT-PCR. The expression of genes associated with pro-inflammatory cytokines and SASP factors, including Illa, Il6, and Tnf, which are upregulated during ageing and enhance matrix degradation [35], were significantly downregulated in Z-IP articular cartilage compared to 
a

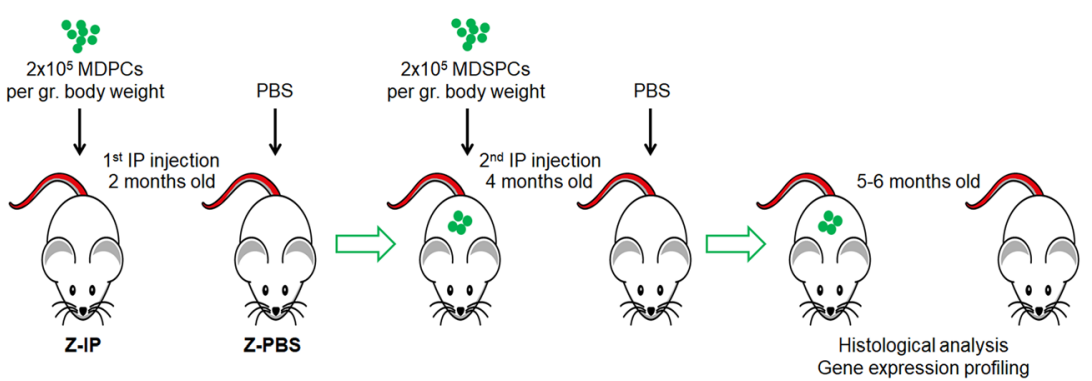

b

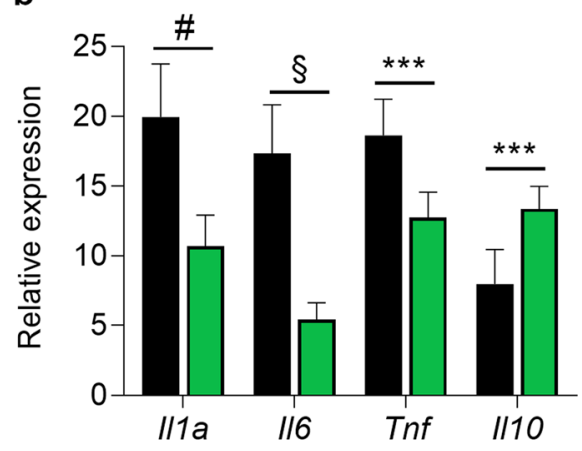

d

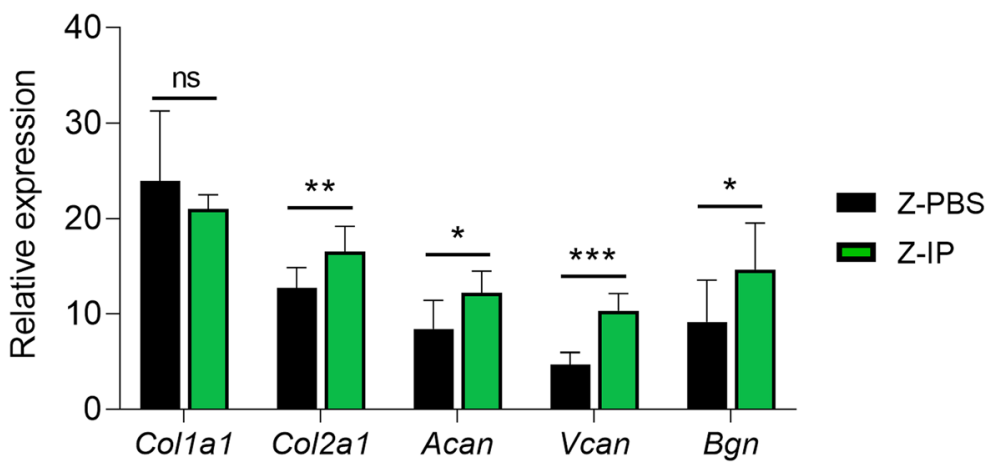

Fig. 2 Effect of Young MDSPC Systemic Transplantation in the Zmpste24-/- Progeroid Mouse Articular Cartilage Microenvironment. a Schematic illustrating the experimental design. b-d Quantitation of pro-inflammatory cytokines and SASP markers (I/1a, II6, and Tnf) and anti-inflammatory cytokine (I/10), extracellular matrix (ECM) proteinases (Mmp3, Mmp13, and Adamts5), and ECM components (Col1a1, Col2a1, Acan, Vcan, and Bgn) relative mRNA levels, measured by qRT-PCR from the knee articular cartilage of Zmpste24 ${ }^{-1-}$ mice intraperitoneally (IP) injected with MDSPCS (Z-IP, $n=7)$ or PBS (Z-PBS, $n=9)$. Expression values are relative to housekeeping genes, Gapdh and Hmgb1. Data are mean \pm SEM. ${ }^{*} p<0.05$, ${ }^{* *} p<0.01$, ${ }^{* * *} p<0.001,{ }^{\#} p<0.0001,{ }^{\$} p<0.00001,{ }^{\ddagger} p<0.000001$, ns: not significant using two-tailed, unpaired Student's t-test or Welch's unequal variance $t$-test

Z-PBS littermates (Fig. 2b). Importantly, the expression of Il10, an immunomodulatory and anti-inflammatory cytokine, was significantly increased in Z-IP mice (Fig. 2b). Gene expression of Mmp3, Mmp13, and Adamts5 were also significantly decreased in articular cartilage of Z-IP mice compared with Z-PBS mice (Fig. 2c). Moreover, Z-IP mice showed significant increases in expression of genes responsible for cartilage mechanical support and tissue repair (Col2a1), cartilage resilience (Acan), chondrocyte proliferation and differentiation (Vcan), and cartilage growth (Bgn) compared to Z-PBS mice (Fig. 2d) [36-39]. Of note, we did not observe a difference in Col1a1 gene expression between treatment groups. These data provide evidence that MDSPC transplantation inhibited genes that promote cartilage degradation while activating pro-regenerative genes, thereby preserving a healthy anabolic-catabolic balance in progeroid AC.

\section{Systemic transplantation of young MDSPCs alters} expression of ageing-related genes in articular cartilage of progeroid mice

We next investigated whether MDSPC transplantation can prevent the changes in expression of key ageingrelated genes in the AC of Zmpste24 $4^{-/-}$progeroid mice (Fig. 3). Our results demonstrate that MDSPC 


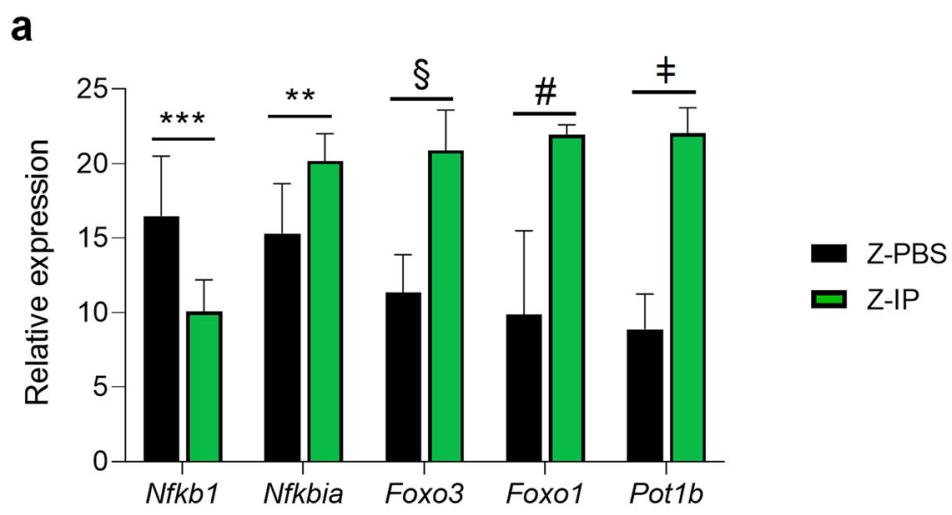

b

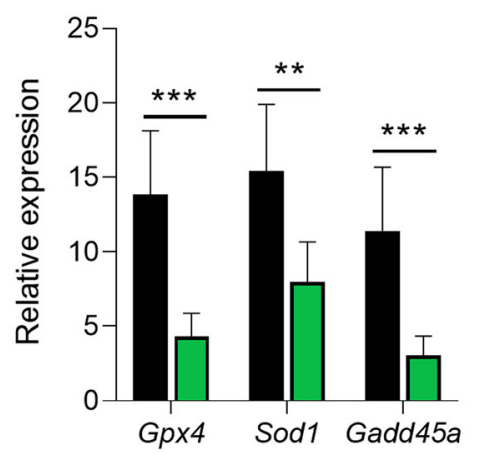

C

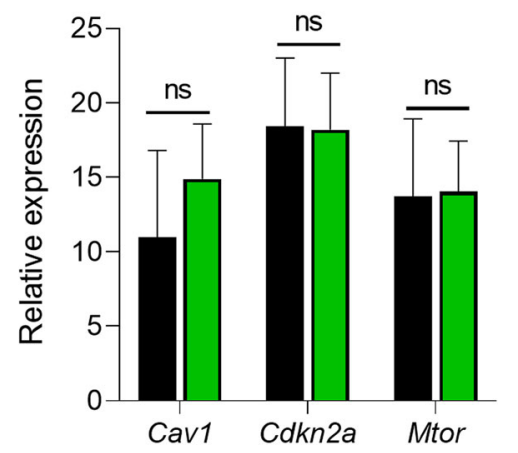

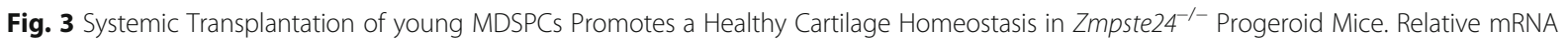
levels were measured by qRT-PCR from the knee articular cartilage of Zmpste24 ${ }^{-1-}$ mice intraperitoneally (IP) injected with MDSPCs (Z-IP, $\left.n=7\right)$ or PBS (Z-PBS, $n=9$ ) and analyzed for the presence of (a) a key ageing signaling pathway mediator (Nfkb1), its inhibitor (Nfkbia), autophagy and prolongevity markers (Foxol and Foxo3), and telomere protection (Pot1b), (b) antioxidant response (Gpx4), oxidative stress-response (Sod1), and growth arrest and DNA damage response (Gadd45a), (c) endocytosis and stress-induced senescence (Cav1), the cellular senescence/tumor suppressor mechanism (Cdkn2a), and the mammalian target of rapamycin (Mtor) genes. Expression values are relative to housekeeping genes, Gapdh and Hmbg1. Data are mean \pm SEM. ${ }^{* *} p<0.01,{ }^{* * *} p<0.001,{ }^{\#} p<0.0001,{ }^{\$} p<0.00001,{ }^{\ddagger} p<0.000001$, ns: not significant using two-tailed, unpaired Student's $t$-test or Welch's unequal variance $t$-test (a-c and e-g)

transplantation significantly down-regulated $N f k b 1$, the master regulator and transcription factor most highly correlated with ageing. Interestingly, the expression level of the NF-kB inhibitor, Nfkbia, was significantly upregulated in Z-IP mice (Fig. 3a) compared to Z-PBS littermates. Our results also revealed a significant increase in the FoxO transcription factors, Foxo3 and Foxo1, in the AC of Z-IP mice compared to Z-PBS mice (Fig. 3a). With age, chondrocytes exhibit senescent phenotypes, including telomere shortening [40]. Notably, the telomere protection gene Pot1b, known as a pro-longevity marker, [41] was robustly increased in the cartilage of ZIP mice (Fig. 3a). MDSPC transplantation significantly decreased the expression level of the oxidative stressresponse gene Sod1, the antioxidant enzyme glutathione peroxidase $4(G p x 4)$, and the growth arrest and DNA damage response gene Gadd45a, compared to Z-PBS mice (Fig. 3b). We did not detect any differences in Mtor-the key regulator and suppressor of autophagy during ageing (Fig. 3c). Also, the expression level of stress-induced senescence gene Cav1 $[42,43]$ - a major component of the caveolae structure and a transmembrane protein, and of cellular senescence gene $C d k n 2 a$, also known as p16 [44], did not change following MDSP $\mathrm{C}$ transplantation (Fig. 3c). Together, these results indicate systemic transplantation of young MDSPCs restores aged articular cartilage homeostasis and improves maintenance at a molecular level.

\section{Systemic transplantation of young MDSPCs rejuvenates articular cartilage in $\mathrm{Zmpste24^{-/ }}$ progeroid mice}

To determine whether MDSPC treatment maintains healthy $\mathrm{AC}$ tissue structure by preventing the loss of ECM proteoglycans, knee joints were stained with Safranin-O (Saf-O). Saf-O staining in the femoral condyle and tibial plateau (Fig. 4a) of the articular cartilage revealed striking changes between Z-IP and Z-PBS mice. In fact, while both groups maintained a similar overall total cartilage area (Fig. 4b), $69 \%$ of the cartilage area in Z-IP mice was Saf-O + compared to only $23 \%$ in Z-PBS 


\section{a}
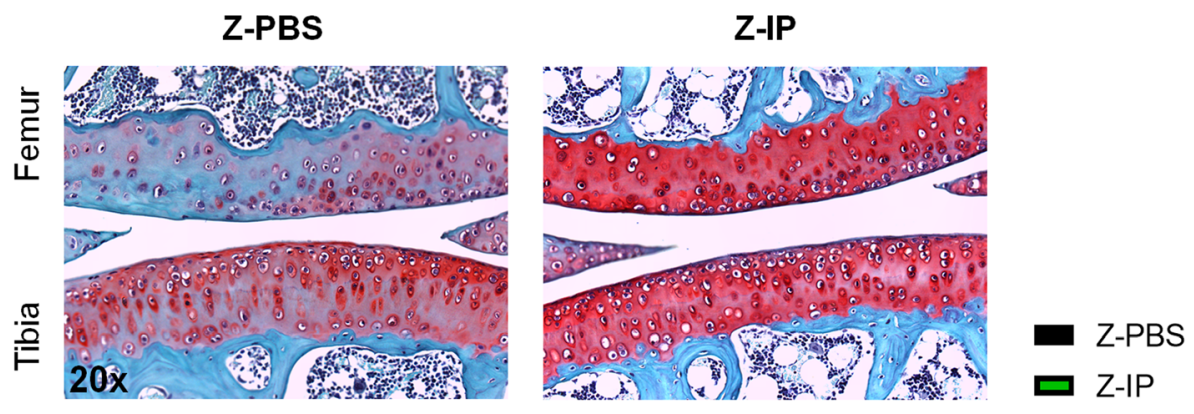

b

C
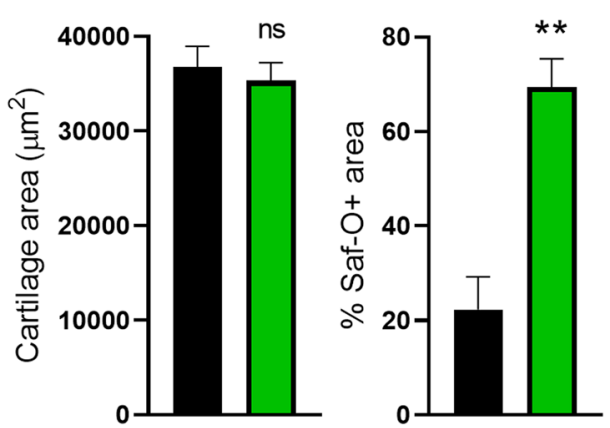

d

e

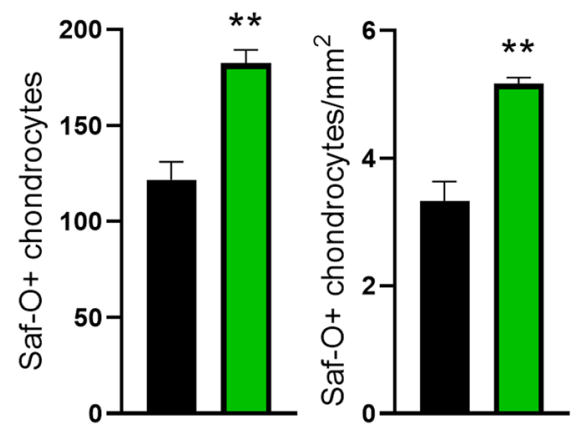

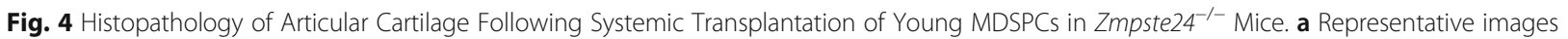
(20x magnification) of Safranin-O (Saf-O) stained femoral condyle and tibial plateau from PBS- (Z-PBS, $n=3$ ) and MDSPC-intraperitoneally transplanted (Z-IP; $n=3) Z$ mpste24 $4^{-/-}$mice at 5-6 months of age. Quantitative histomorphometric analysis shows (b) total cartilage area, (c) percent of Saf-O + cartilage area, (d) chondrocyte number, and (e) chondrocyte density. Data are mean \pm SEM. ${ }^{*} p<0.05$ with two-tailed, unpaired Student's t-test

mice (Fig. 4c), indicating proteoglycan enrichment after MDSPC transplantation. The knee joints of Z-IP mice also had a greater number of chondrocytes and density compared to Z-PBS control mice (Fig. 4d and e). Together, these findings strongly suggest that MDSPC treatment confers protection against the loss of $\mathrm{AC}$ matrix, in part by increasing cartilage cellularity and preventing age-associated degradation.

To investigate how young MDSPCs rejuvenate aged $\mathrm{AC}$, we analyzed the $\mathrm{AC}$ throughout the knee joint for donor cell engraftment. MDSPCs were transduced with a retroviral vector containing a nuclear localization sequence ( $n L a c Z)$ prior to transplantation. As a positive control for tracking donor cells, transduced MDSPCs were also intramuscularly (IM) transplanted into gastrocnemius muscles of WT control mice. Large numbers of $L a c Z+$ MDSPCs were detected in the control IM injected muscle, however, no donor MDSPCs were detected in the AC-or surrounding tissues-of any Z-IP mouse (Supplementary Fig. S1). This indicates that the beneficial effects are not due to direct tissue or chondrocyte reconstitution by donor MDSPCs, but are rather mediated through their therapeutic secreted factors that act systemically.

\section{Discussion}

Given that AC degeneration causes OA, a significant cause of disability worldwide, we sought to identify agerelated changes in the cartilage microenvironment in a mouse model of accelerated ageing. Furthermore, we evaluated an intervention to delay or reverse the degenerative effects of accelerated ageing. Here, we report, for the first time, the prevention of progeroid $\mathrm{AC}$ degeneration-at the molecular and tissue levels-by systemic transplantation of young WT MDSPCs.

Zmpste24 ${ }^{-/-}$mice exhibit gene expression signatures robustly associated with ageing in $\mathrm{AC}-$ such as increased SASP factors including pro-inflammatory cytokines (II1 $a$ and Il6) and catabolic matrix-degrading proteinases (Mmp3, Mmp13, and Adamts5), which further drive age-associated pathologies such as hypertrophic chondrocyte differentiation. However, after MDSPC treatment, Zmpste $24^{-/-}$gene expression profiles remained similar to those of WT littermates [12, 37]. Furthermore, the immunomodulating and antiinflammatory cytokine $I l 10$ and an ECM component that plays an important role in tissue remodeling and repair, Col2a1 [38], were upregulated in the AC of Z-IP mice. Rejuvenation was also supported by increased expression 
of vital matrix proteoglycans Acan, Vcan, and Bgn, which are responsible for cartilage tensile strength, resilience, and cartilage growth. Together, these results demonstrate the restoration of progeroid cartilage to a "healthy" state.

The fact that systemic transplantation provides such a remarkable improvement in progeroid $\mathrm{AC}$, despite the fact that no donor cells could be detected in AC, reinforces our hypothesis that the observed effects are induced via secreted paracrine/endocrine factors. This is consistent with our previous reports [22, 25] where young (but not old) MDSPCs restored the dysfunction of progeroid and naturally aged MDSPCs when cocultured in vitro, and that transplantation of young MDSPCs extended both the lifespan and healthspan of progeroid mice through systemically acting secreted factors. Indeed, more recently, we have also shown that systemic therapy with young MDSPCs results in restoration of naturally aged (2-yr old) articular cartilage-at the molecular, tissue, and functional levels [26]. Similarly, no transplanted cells were found in $\mathrm{AC}$, however, systemic transplantation of MDSPC reduced inflammatory factors and ECM proteinases, increased anabolic factors and proteoglycan content, and induced the expression of pro-regenerative and pro-longevity genes. As a result, the MDSPC-treated naturally aged mice exhibited $\mathrm{AC}$ regeneration and significant functional improvement [26]. The underlying mechanism(s) of action for this rejuvenation still remains unknown, but our ongoing studies focus on the mechanistic basis of MDSPC systemic effects. We hypothesize that MDSPCs promote the maintenance of healthy AC by secreting chondrocyte-protecting cytokines/growth factors, thereby regulating chondrocyte viability and sensitivity to extrinsic factors and reducing articular cartilage degeneration. In fact, MDSPCs are known to perform a variety of biological functions, including immune regulation, angiogenesis, anti-apoptosis, anti-oxidant, cell homing, and promotion of tissue-specific cell differentiation [45]. Therefore, it is not far-fetched to suggest that proregenerative factors secreted by MDSPCs preserves the local $\mathrm{AC}$ microenvironment in progeroid mice by increasing anabolic activity while simultaneously inhibiting catabolic activity.

The fact that the DNA damage-inducible and oxidative stress genes Gadd45a, Sod1, Gpx4, were all significantly decreased following systemic transplantation, indicates that MDSPCs may also preserve AC through cytoprotective mechanisms. This is supported by the observation that we measured a profound increase in expression of the telomere protection gene, Pot $1 b$, demonstrating a protective effect of MDSPCs on chromosomal stability. In fact, recent studies have shown that maintenance of telomere length can reverse neurodegeneration, delay metabolic ageing in mice, and increase lifespan $[41,46]$.

The transcription factor NF- $\mathrm{kB}$, a central controller and mediator of ageing-related signaling pathways, regulates many immune responses [47] and is also a candidate activator of many ageing-related transcriptional changes in human and mouse tissues, particularly stressinducing stimuli such as pro-inflammatory factors and SASPs [48]. Previous studies by Robbins et al. indicate that $N f k b 1$ transcriptional activity is up-regulated in a variety of tissues in both natural ageing and in mouse models of human progeria caused by defective DNA damage repair mechanisms [49]. Skin-derived human fibroblasts from HGPS patients and aged individuals also show increased inflammatory gene expression and increased levels of NF- $\mathrm{kB}$ activation compared to cells from young individuals $[48,50]$. Similar to previous reports [51], systemic transplantation of MDSPCs results in significant reduction of $N f k b 1$ expression and an increase in expression of the Nfkb1 inhibitor, Nfkbia. Further studies are needed to investigate the mechanism(s) underlying modulation of NF-kB and other inflammatory factors.

Defective autophagy is a key feature of age-related diseases, and recent observations indicate that this process is compromised in ageing cartilage [52, 53] through FoxO downregulation [54]. FoxO transcription factors, which have recently been found to play vital roles in postnatal cartilage development, maturation, homeostasis, and protection against $\mathrm{OA}$-associated cartilage damage [54] as well as to protect against cellular ageing and to enhance autophagy, are suppressed in human OA and mouse cartilage [11, 55]. Foxo1 and Foxo3 regulate chondrocyte proliferation and differentiation [54] and their expression levels are reduced in both ageing and OAaffected cartilage in humans and mice [54]. In our study, the prominent upregulation of Foxo1 and Foxo3 in AC in MDSPC-treated mice suggests that our cellular therapy prevents chondrocyte ageing and delays the onset of OA-like symptoms, in part, through these mechanisms. It appears that, by preserving cartilage cellularity, the bioactive paracrine/endocrine factors secreted by MDSP Cs had beneficial effects on the aged cartilage tissue microenvironment, leading to more favorable conditions for tissue regeneration. This results in increased chondrocyte density and an enrichment of ECM proteoglycans, thereby maintaining more normal knee joint histology.

Collectively, our results suggest that the mechanisms behind $\mathrm{AC}$ rejuvenation in progeroid mice are in part due to activation of telomere protection mechanisms, modulation of the inflammatory SASP cascade, and maintenance of pro-regenerative and pro-longevity 
pathways. Further studies will identify MDSPC secretomes, specifically those which are activated after IP transplantation. Limitations of this study include that we did not assess functional effects of MDSPC transplantation or possible effects of sex differences between donor and host. Future studies will investigate if the observed restoration of $\mathrm{AC}$ at the molecular and cellular levels after MDSPC systemic transplantation lead to functional stability or improvement in progeroid mice.

\section{Conclusions}

These results demonstrate that treating a mouse model of accelerated ageing with a systemic injection of young multipotent adult stem cells prevents age-related cartilage degradation and maintains the catabolic/anabolic balance in host tissues. This cellular treatment promotes a "healthy" homeostasis and stimulates tissue regeneration. Young MDSPCs, and/or their secreted factors, thus represent a potential novel therapeutic strategy for preventing or treating age-related AC degeneration.

\section{Methods \\ MDSPC isolation}

Young WT MDSPCs were isolated from the hindlimb skeletal muscle of 21 day-old female mice via a modified preplate technique [18]. MDSPCs were cultured and expanded for transplantation in proliferation medium (PM): Hi-glucose DMEM supplemented with $10 \%$ horse serum, $10 \%$ fetal-bovine serum (FBS), $1 \%$ penicillinstreptomycin (all from Invitrogen), and $0.5 \%$ chick embryo extract (CEE, Accurate Chemical). Cells are cultured on collagen type I-coated flasks.

\section{Animal Husbandry}

All animal experiments were performed with the approval of the Northwestern University Institutional Animal Care and Use Committee. 17-day-old mice are ear tagged and tail snips are collected and mailed through the Northwestern University in-house system to Transnetyx for genotyping. Results are received within $72 \mathrm{~h}$ with over $99.9 \%$ accuracy. Primers and PCR conditions for genotyping Zmpste24 ${ }^{-1-}$ mice have been previously published [30]. Mice were maintained in a pathogen-free facility at $23-24{ }^{\circ} \mathrm{C}$ under a 12-hr light, 12-hr dark regimen and fed ad libitum a standard chow which is gamma-irradiated. Mice were always studied in sibling pairs to minimize environmental variables. Only if breeding produces two or more Zmpste24 $4^{-1-}$ mice would the litter be used in an exposure study so that one mutant animal is treated with the experimental treatment and the other receives vehicle only.

\section{Cell Transplantation}

MDSPCs suspended in $50 \mu \mathrm{L}$ of buffered saline (PBS) were transplanted via intraperitoneal (IP) administration into 4-5-monthold Zmpste24 $4^{-/-}$mice at $2 \times 10^{5}$ MDSP Cs per gram body-weight. A littermate mutant animal was injected with vehicle only (PBS). The injection was repeated 2 months later. Mice were sacrificed at 5-6 months of age and the right hind limbs were harvested for histopathological analysis and the left knee joint were isolated for gene expression analysis. Both male and female mice were included in the transplantation study.

\section{Histology}

Right hind limbs were fixed in PFA for 2 days, stored in PBS at $4^{\circ} \mathrm{C}$ overnight, and then paraffin embedded. Sections were cut to $4 \mu \mathrm{m}$, collected at $100 \mu \mathrm{m}$ intervals, and stained with Safranin O (Saf-O)-Fast Green as described previously to evaluate the proteoglycan content and pathological changes such as cartilage degradation [56]. To detect $n L a c Z$ expression, paraffin embedded sections of AC were deparaffinized in serial washes of xylene and rehydrated in sequential washes of 100-70\% ethanol. The tissue sections were then incubated with $\mathrm{X}$ gal solution overnight at room temperature and counterstained with eosin.

\section{Histomorphometry}

Histomorphometric analyses were performed using NISElements software (Nikon, AR 5.11.03). The Saf-O + area was measured impartially by the NIS software using identical thresholding parameters between all images (pixels falling below the threshold intensity of 20 were uncounted) by detecting the total red (Saf-O+) pixel area. The total cartilage area was manually selected with border detection assistance using the NIS software's thresholding tools. Saf-O + chondrocytes were manually counted for each femoral condyle and tibial plateau and graphed as average Saf-O + chondrocytes and number of Saf-O + chondrocytes per $\mathrm{mm}^{2}$ of total articular cartilage. Chondrocytes were considered $\mathrm{Saf}-\mathrm{O}+$ if they were surrounded by red-stained matrix.

\section{RNA isolation and qPCR}

To measure mRNA expression, total RNA was extracted from articular cartilage isolated from paraformaldehyde (PFA)-fixed knee joints using an RNeasy ${ }^{\circ}$ mini kit for formalin-fixed, paraffin-embedded (FFPE) tissue (Qiagen) according to manufacturer's protocol. RNA quality was validated using an Eppendorf BioSpectrophotometer, and $100 \mathrm{ng}$ of total RNA was reverse-transcribed according to the manufacture's protocol using iScript Advanced cDNA synthesis kit (Bio-Rad Laboratories). Pre-amplification of the primers was carried out according to the manufacturer's protocol 
Table 1 Primer list

\begin{tabular}{|c|c|c|}
\hline Gene name & $\begin{array}{l}\text { Traditional classification } \\
\text { (Common name) }\end{array}$ & Catalog identification \\
\hline Acan & Aggrecan & qMmuCED0046843 \\
\hline Adamts5 & a-disintegrin and metalloproteinase with thrombospondin motif 5 & qMmuCED0045481 \\
\hline Bgn & Biglycan & qMmuCED0046901 \\
\hline Cav1 & Caveolin 1 & qMmuCID0020997 \\
\hline$C d k n 2 a$ & Cyclin dependent kinase inhibitor $2 a$ & qMmuCED0038108 \\
\hline Collar & Collagen type I alpha 1 & qMuCED0044222 \\
\hline Col2ar & Collagen type 2 a1 & qMuCID0006546 \\
\hline Colloar & Collagen type 10 alpa1 & qMuCID0008115 \\
\hline Foxo1 & Fork head box transcription factor 1 & qMmuCID0016391 \\
\hline Foxo3 & Fork head box transcription factor 3 & qMmuCED0004522 \\
\hline Gadd45a & Growth arrest and DNA damage inducible alpha & qMmuCED0001074 \\
\hline Gapdh & Glyceraldehyde 3-phosphate dehydrogenase & qMmuCED0027497 \\
\hline Gpx4 & Glutathione peroxidase & qMmuCED0001062 \\
\hline Hmgbl & High mobility group box 1 & qMmuCE00041193 \\
\hline $117 a$ & Interleukin 1 alpha & qMmuCID005637 \\
\hline 116 & Interleukin 6 & qMmuCED0045760 \\
\hline 1110 & Interleukin 10 & qMmuCED0044967 \\
\hline Mmp3 & Matrix metalloproteinase 3 & qMmuCED0049170 \\
\hline Mmp13 & Matrix metalloproteinase 13 & qMmuCED0050490 \\
\hline Mtor & Mammalian target of rapamycin & qMmuCED0047795 \\
\hline Nfkb1 & Nuclear factor kappa b1 & qMmuCID0005357 \\
\hline Nfkbia & Nuclear factor kappa b inhibitor & qMmuCED0045043 \\
\hline Pot1b & Protection of telomeres $1 \mathrm{~b}$ & qMmuCED0045942 \\
\hline Sod1 & Superoxide dismutase 1 & qMmuCID0026086 \\
\hline Tnf & Tumor necrosis factor & qMmuCED004141 \\
\hline Vcan & Versican & qMmuCED0046650 \\
\hline
\end{tabular}

using a Pre-amplification kit (Bio-Rad Laboratories). The pre-amplified cDNA was diluted and used for analysis of gene expression changes in $10 \mu \mathrm{L}$ reactions using SYBR green advanced master mix kit (Bio-Rad Laboratories) and the gene of interest primer pairs. Data were analyzed with the $\Delta \mathrm{Ct}$ method and gene expression was normalized to average expression of housekeeping genes, Gapdh and Hmgb1. Primers for the genes of interest were obtained from Bio-Rad Laboratories and the primer catalog identification IDs are listed in Table 1.

\section{Statistics}

Statistical analyses were carried out using the SigmaPlot (Jandel Scientific v14.0) software package. The two-tailed unpaired Welch's unequal variance $t$-test, two-tailed Student $t$-test, or the Mann-Whitney Rank sum test were used where appropriate for direct comparisons between treatment and control groups. All values are expressed as the mean \pm SEM., and $p<0.05$ was considered significant.

\section{Supplementary Information}

The online version contains supplementary material available at https://doi. org/10.1186/s12979-021-00239-8.

Additional file 1: Supplementary Figure S1. Determination of donor cell engraftment after transplantation of $Z$ mpste $24^{-/}$mice with young MDSPCs. Articular cartilage of Zmpste24 $4^{-/-}$mice (Z-IP) intraperitoneally injected with $2 \times 10^{5}$ MDSPCs transduced to express a nuclear LacZreporter gene for donor cell tracking, were stained with $X$-gal and Eosin (pink) to determine sites of engraftment. Gastrocnemius muscles of 6-month old wildtype (WT) mice intramuscularly (IM) injected with the same MDSPCs were identically stained as a positive control. Brightfield images show donor cells (LacZ+, blue) were not detected in articular cartilage, however, as expected, large numbers of donor cells were detected in gastrocnemius muscles of control IM injected WT mice (top row seen at $5 x$ magnification and bottom row seen at 10x magnification).

\section{Acknowledgements}

We would like to thank Dr. Carlos Lopez-Otin for kindly providing the breeding pairs of Zmpste24-1- mice from University of Oviedo, Spain, Katherine Gruner and Alicia Steffens at the Northwestern Mouse Histology and Phenotyping Laboratory for their expertise and assistance processing the articular cartilage tissues, and Dr. Ann Barlow for her editorial assistance. 


\section{Authors' contributions}

S.D.T. contributed to experimental design, cell transplantation, microscopy imaging of cartilage tissues, histological analysis, and prepared the manuscript. R.P. contributed to experimental design, QRT-PCR, genetic analysis, and histological analysis. R.L.L. contributed to experimental design and manuscript editing. M.L. conceived and oversaw all experiments, performed cell transplantation, data interpretation, statistical analysis, and edited the manuscript. All authors critically revised and approved the final version of the manuscript.

\section{Funding}

This work was supported by the Christopher L. Moseley Foundation, the Lisa Dean Moseley Foundation, and an Innovative Catalyst Grant Award from Shirley Ryan AbilityLab to M.L. and a Research Career Scientist Award, Number IK6 RX003351, from the United States Department of Veterans Affairs Rehabilitation R\&D Service to R.L.L.

\section{Availability of data and materials}

The datasets used and/or analyzed during the current study are available from the corresponding author on reasonable request.

\section{Declarations}

\section{Ethics approval and consent to participate}

Not applicable.

\section{Consent for publication}

Not applicable.

\section{Competing interests}

The authors declare that they have no competing interests.

\section{Author details}

'Shirley Ryan Abilitylab (Formerly the Rehabilitation Institute of Chicago), 355 E. Erie St, IL 60611 Chicago, USA. ${ }^{2}$ Department of Physical Medicine and Rehabilitation, Northwestern University, Chicago, USA. ${ }^{3}$ Northwestern University Interdepartmental Neuroscience (NUIN) Graduate Program, Northwestern University, Chicago, USA.

\section{Received: 17 August 2020 Accepted: 26 May 2021}

Published online: 07 June 2021

\section{References}

1. Wells T, Davidson C, Morgelin M, Bird JL, Bayliss MT, Dudhia J. Age-related changes in the composition, the molecular stoichiometry and the stability of proteoglycan aggregates extracted from human articular cartilage. Biochem J. 2003;370(Pt 1):69-79.

2. Verzijl N, DeGroot J, Ben ZC, Brau-Benjamin O, Maroudas A, Bank RA, et al. Crosslinking by advanced glycation end products increases the stiffness of the collagen network in human articular cartilage: a possible mechanism through which age is a risk factor for osteoarthritis. Arthritis Rheum. 2002; 46(1):114-23.

3. Lawrence RC, Helmick CG, Arnett FC, Deyo RA, Felson DT, Giannini EH, et al. Estimates of the prevalence of arthritis and selected musculoskeletal disorders in the United States. Arthritis Rheum. 1998:41(5):778-99.

4. DALYs GBD, Collaborators H. Global, regional, and national disabilityadjusted life-years (DALYs) for 333 diseases and injuries and healthy life expectancy (HALE) for 195 countries and territories, 1990-2016: a systematic analysis for the Global Burden of Disease Study 2016. Lancet. 2017. 390(10100):1260-344.

5. Soto-Gamez A, Demaria M. Therapeutic interventions for aging: the case of cellular senescence. Drug Discov Today. 2017;22(5):786-95.

6. Greene MA, Loeser RF. Aging-related inflammation in osteoarthritis. Osteoarthr Cartil. 2015;23(11):1966-71

7. Freund A, Orjalo AV, Desprez PY, Campisi J. Inflammatory networks during cellular senescence: causes and consequences. Trends Mol Med. 2010;16(5): 238-46

8. Loeser RF. Aging and osteoarthritis: the role of chondrocyte senescence and aging changes in the cartilage matrix. Osteoarthritis Cartilage. 2009; 17(8):971-9.

9. Loeser RF. Aging and osteoarthritis. Curr Opin Rheumatol. 2011;23(5):492-6.
10. Terman A, Kurz T, Navratil M, Arriaga EA, Brunk UT. Mitochondrial turnover and aging of long-lived postmitotic cells: the mitochondriallysosomal axis theory of aging. Antioxid Redox Signal. 2010;12(4):50335.

11. Carames B, Taniguchi N, Otsuki S, Blanco FJ, Lotz M. Autophagy is a protective mechanism in normal cartilage, and its aging-related loss is linked with cell death and osteoarthritis. Arthritis Rheum. 2010;62(3):791801.

12. Lotz M, Loeser RF. Effects of aging on articular cartilage homeostasis. Bone. 2012:51(2):241-8.

13. Krill M, Early N, Everhart JS, Flanigan DC. Autologous Chondrocyte Implantation (ACI) for knee cartilage defects: a review of indications, technique, and outcomes. JBJS Rev. 2018;6(2):e5

14. Davatchi F, Sadeghi Abdollahi B, Mohyeddin M, Nikbin B. Mesenchymal stem cell therapy for knee osteoarthritis: 5 years follow-up of three patients. Int J Rheum Dis. 2016;19(3):219-25.

15. Al-Najar M, Khalil H, Al-Ajlouni J, Al-Antary E, Hamdan M, Rahmeh R, et al. Intra-articular injection of expanded autologous bone marrow mesenchymal cells in moderate and severe knee osteoarthritis is safe: a phase I/II study. J Orthop Surg Res. 2017;12(1):190.

16. Bartlett W, Skinner JA, Gooding CR, Carrington RW, Flanagan AM, Briggs TW, et al. Autologous chondrocyte implantation versus matrix-induced autologous chondrocyte implantation for osteochondral defects of the knee: a prospective, randomised study. J Bone Joint Surg Br. 2005;87(5): 640-5.

17. Krajewska-Wlodarczyk M, Owczarczyk-Saczonek A, Placek W, Osowski A, Wojtkiewicz J. Articular cartilage aging-potential regenerative capacities of cell manipulation and stem cell therapy. Int J Mol Sci. 2018;19(2):623.

18. Lavasani M, Lu A, Thompson SD, Robbins PD, Huard J, Niedernhofer LJ. Isolation of muscle-derived stem/progenitor cells based on adhesion characteristics to collagen-coated surfaces. Methods Mol Biol. 2013;976:5365.

19. Lavasani M, Lu A, Peng H, Cummins J, Huard J. Nerve growth factor improves the muscle regeneration capacity of muscle stem cells in dystrophic muscle. Hum Gene Ther. 2006;17(2):180-92

20. Deasy BM, Gharaibeh BM, Pollett JB, Jones MM, Lucas MA, Kanda Y, et al. Long-term self-renewal of postnatal muscle-derived stem cells. Mol Biol Cell. 2005;16(7):3323-33.

21. Vella JB, Thompson SD, Bucsek MJ, Song M, Huard J. Murine and human myogenic cells identified by elevated aldehyde dehydrogenase activity: implications for muscle regeneration and repair. PLoS One. 2011;6(12): e29226.

22. Lavasani M, Robinson AR, Lu A, Song M, Feduska JM, Ahani B, et al. Musclederived stem/progenitor cell dysfunction limits healthspan and lifespan in a murine progeria model. Nat Commun. 2012;3:608.

23. Adachi N, Sato K, Usas A, Fu FH, Ochi M, Han CW, et al. Muscle derived, cell based ex vivo gene therapy for treatment of full thickness articular cartilage defects. J Rheumatol. 2002;29(9):1920-30.

24. Kuroda R, Usas A, Kubo S, Corsi K, Peng H, Rose T, et al. Cartilage repair using bone morphogenetic protein 4 and muscle-derived stem cells. Arthritis Rheum. 2006;54(2):433-42.

25. Song M, Lavasani M, Thompson SD, Lu A, Ahani B, Huard J. Musclederived stem/progenitor cell dysfunction in Zmpste24-deficient progeroid mice limits muscle regeneration. Stem Cell Res Ther. 2013; 4(2):33.

26. Thompson SD, Pichika R, Lieber RL, Budinger GRS, Lavasani M. Systemic transplantation of adult multipotent stem cells functionally rejuvenates aged articular cartilage. Aging Dis Published online November 18, 2020; 12(3). doi:https://doi.org/10.14336/AD.2020.1118.

27. Leung GK, Schmidt WK, Bergo MO, Gavino B, Wong DH, Tam A, et al. Biochemical studies of Zmpste24-deficient mice. J Biol Chem. 2001;276(31): 29051-8.

28. Greising SM, Call JA, Lund TC, Blazar BR, Tolar J, Lowe DA. Skeletal muscle contractile function and neuromuscular performance in Zmpste24 (-/-) mice, a murine model of human progeria. Age (Dordr) 2012;34(4):805-19.

29. Bergo MO, Gavino B, Ross J, Schmidt WK, Hong C, Kendall LV, et al. Zmpste24 deficiency in mice causes spontaneous bone fractures, muscle weakness, and a prelamin A processing defect. Proc Natl Acad Sci U S A. 2002;99(20):13049-54 
30. Pendas AM, Zhou Z, Cadinanos J, Freije JM, Wang J, Hultenby K, et al Defective prelamin A processing and muscular and adipocyte alterations in Zmpste24 metalloproteinase-deficient mice. Nat Genet. 2002;31(1):94-9.

31. Badame AJ. Progeria. Arch Dermatol. 1989;125(4):540-4

32. Chuchana P, Mausset-Bonnefont AL, Mathieu M, Espinoza F, Teigell M, Toupet K, et al. Secreted alpha-Klotho maintains cartilage tissue homeostasis by repressing NOS2 and ZIP8-MMP13 catabolic axis. Aging. 2018;10(6):144253.

33. Wu W, Billinghurst RC, Pidoux I, Antoniou J, Zukor D, Tanzer M, et al. Sites of collagenase cleavage and denaturation of type II collagen in aging and osteoarthritic articular cartilage and their relationship to the distribution of matrix metalloproteinase 1 and matrix metalloproteinase 13. Arthritis Rheum. 2002:46(8):2087-94

34. Glasson SS, Askew R, Sheppard B, Carito B, Blanchet T, Ma HL, et al. Deletion of active ADAMTS5 prevents cartilage degradation in a murine model of osteoarthritis. Nature. 2005;434(7033):644-8.

35. Loeser RF, Olex AL, McNulty MA, Carlson CS, Callahan MF, Ferguson CM, et al. Microarray analysis reveals age-related differences in gene expression during the development of osteoarthritis in mice. Arthritis Rheum. 2012; 64(3):705-17.

36. Choocheep K, Hatano S, Takagi H, Watanabe H, Kimata K, Kongtawelert P, et al. Versican facilitates chondrocyte differentiation and regulates joint morphogenesis. J Biol Chem. 2010;285(27):21114-25.

37. Peffers M, Liu X, Clegg P. Transcriptomic signatures in cartilage ageing. Arthritis Res Ther. 2013;15(4):R98.

38. Stoop R, van der Kraan PM, Buma P, Hollander AP, Billinghurst RC, Poole AR, et al. Type II collagen degradation in spontaneous osteoarthritis in C57BI/6 and BALB/C mice. Arthritis Rheum. 1999;42(11):2381-9.

39. Roughley PJ, Melching LI, Recklies AD. Changes in the expression of decorin and biglycan in human articular cartilage with age and regulation by TGFbeta. Matrix Biol. 1994;14(1):51-9.

40. Martin JA, Buckwalter JA. The role of chondrocyte senescence in the pathogenesis of osteoarthritis and in limiting cartilage repair. J Bone Joint Surg Am. 2003;85-A Suppl 2:106-10.

41. Munoz-Lorente MA, Cano-Martin AC, Blasco MA. Mice with hyper-long telomeres show less metabolic aging and longer lifespans. Nat Commun. 2019;10(1):4723.

42. Nguyen KC, Cho KA. Versatile functions of Caveolin-1 in aging-related diseases. Chonnam Med J. 2017;53(1):28-36.

43. Min TU, Sheng LY, Chao C, Jian T, Guang GS, Hua LG. Correlation between osteopontin and caveolin-1 in the pathogenesis and progression of osteoarthritis. Exp Ther Med. 2015;9(6):2059-64.

44. Campisi J, d'Adda di Fagagna F. Cellular senescence: when bad things happen to good cells. Nat Rev Mol Cell Biol. 2007;8(9):729-40.

45. Gharaibeh $\mathrm{B}$, Lavasani M, Cummins $\mathrm{JH}$, Huard J. Terminal differentiation is not a major determinant for the success of stem cell therapy - cross-talk between muscle-derived stem cells and host cells. Stem Cell Res Ther. 2011; 2(4):31.

46. Jaskelioff M, Muller FL, Paik JH, Thomas E, Jiang S, Adams AC, et al. Telomerase reactivation reverses tissue degeneration in aged telomerasedeficient mice. Nature. 2011;469(7328):102-6.

47. Gosselin K, Abbadie C. Involvement of Rel/NF-kappa B transcription factors in senescence. Exp Gerontol. 2003;38(11-12):1271-83.

48. Adler AS, Sinha S, Kawahara TL, Zhang JY, Segal E, Chang HY. Motif module map reveals enforcement of aging by continual NF-kappaB activity. Genes Dev. 2007;21(24):3244-57.

49. Tilstra JS, Clauson CL, Niedernhofer LJ, Robbins PD. NF-kappaB in aging and disease. Aging Dis. 2011;2(6):449-65.

50. Kriete A, Mayo KL, Yalamanchili N, Beggs W, Bender P, Kari C, et al. Cell autonomous expression of inflammatory genes in biologically aged fibroblasts associated with elevated NF-kappaB activity. Immun Ageing. 2008:5:5.

51. Adler AS, Kawahara TL, Segal E, Chang HY. Reversal of aging by NFkappaB blockade. Cell Cycle. 2008;7(5):556-9.

52. Rubinsztein DC, Marino G, Kroemer G. Autophagy and aging. Cell. 2011; 146(5):682-95.

53. Lotz MK, Carames B. Autophagy and cartilage homeostasis mechanisms in joint health, aging and OA. Nat Rev Rheumatol. 2011;7(10):579-87.

54. Matsuzaki T, Alvarez-Garcia O, Mokuda S, Nagira K, Olmer M, Gamini R et al. FoxO transcription factors modulate autophagy and proteoglycan
4 in cartilage homeostasis and osteoarthritis. Sci Transl Med. 2018 10(428):eaan0746.

55. Akasaki Y, Hasegawa A, Saito M, Asahara H, Iwamoto Y, Lotz MK. Dysregulated FOXO transcription factors in articular cartilage in aging and osteoarthritis. Osteoarthr Cartil. 2014;22(1):162-70.

56. Kwok J, Onuma H, Olmer M, Lotz MK, Grogan SP, D'Lima DD. Histopathological analyses of murine menisci: implications for joint aging and osteoarthritis. Osteoarthr Cartil. 2016;24(4):709-18.

\section{Publisher's Note}

Springer Nature remains neutral with regard to jurisdictional claims in published maps and institutional affiliations.
Ready to submit your research? Choose BMC and benefit from:

- fast, convenient online submission

- thorough peer review by experienced researchers in your field

- rapid publication on acceptance

- support for research data, including large and complex data types

- gold Open Access which fosters wider collaboration and increased citations

- maximum visibility for your research: over $100 \mathrm{M}$ website views per year

At BMC, research is always in progress.

Learn more biomedcentral.com/submissions 